\title{
Lettuce growth stage identification based on phytomorphological variations using coupled color superpixels and multifold watershed transformation
}

\author{
Ronnie Sabino Concepcion II a,1, ${ }^{\text {, }}$, Jonnel Dorado Alejandrino a,2, Sandy Cruz Lauguico a,3, \\ Rogelio Ruzcko Tobias a,4, Edwin Sybingco a,5, Elmer Pamisa Dadios b,6, Argel Alejandro Bandala b,7 \\ ${ }^{a}$ Electronics and Communications Engineering Department, De La Salle University, Manila, Philippines \\ ${ }^{b}$ Manufacturing Engineering and Management Department, De La Salle University, Manila, Philippines \\ ${ }^{1}$ ronnie_concepcionii@dlsu.edu.ph; ${ }^{2}$ jonnel_alejandrino@dlsu.edu.ph; ${ }^{3}$ sandy_lauguico@dlsue.edu.ph; ${ }^{4}$ rogelio_tobias@dlsu.edu.ph; \\ ${ }^{5}$ edwin.sybingco@dlsu.edu.ph; ${ }^{6}$ elmer.dadios@dlsu.edu.ph; ${ }^{7}$ argel.bandala@dlsu.edu.ph \\ * corresponding author
}

ARTICLE INFO

\section{Article history}

Received December 8, 2019

Revised March 3, 2020

Accepted April 4, 2020

Available online November 13, 2020

\section{Keywords}

Lettuce

Machine learning

Morphological

Superpixels

Watershed

\section{ABSTRACT}

Identifying the plant's developmental growth stages from seed leaf is crucial to understand plant science and cultivation management deeply. An efficient vision-based system for plant growth monitoring entails optimum segmentation and classification algorithms. This study presents coupled color-based superpixels and multifold watershed transformation in segmenting lettuce plant from complicated background taken from smart farm aquaponic system, and machine learning models used to classify lettuce plant growth as vegetative, head development and for harvest based on phytomorphological profile. Morphological computations were employed by feature extraction of the number of leaves, biomass area and perimeter, convex area, convex hull area and perimeter, major and minor axis lengths of the major axis length the dominant leaf, and length of plant skeleton. Phytomorphological variations of biomass compactness, convexity, solidity, plant skeleton, and perimeter ratio were included as inputs of the classification network. The extracted Lab color space information from the training image set undergoes superpixels overlaying with 1,000 superpixel regions employing $\mathrm{K}$-means clustering on each pixel class. Six-level watershed transformation with distance transformation and minima imposition was employed to segment the lettuce plant from other pixel objects. The accuracy of correctly classifying the vegetative, head development, and harvest growth stages are $88.89 \%, 86.67 \%$, and $79.63 \%$, respectively. The experiment shows that the test accuracy rates of machine learning models were recorded as $60 \%$ for LDA, $85 \%$ for ANN, and 88.33\% for QSVM. Comparative analysis showed that QSVM bested the performance of optimized LDA and ANN in classifying lettuce growth stages. This research developed a seamless model in segmenting vegetation pixels, and predicting lettuce growth stage is essential for plant computational phenotyping and agricultural practice optimization.

This is an open access article under the CC-BY-SA license.

\section{Introduction}

Phytomorphology or plant morphology is the science of external structure, physical shape and form, and plants' development. It is comparative, somatically observative, especially on vegetative plant structures. It is concerned with analyzing ultrastructure that ranges from cytology to plant growth habit and its overall architecture, examining plant development. Plant morphology is valuable and useful in visually discriminating the classes of plants. The modern continuum morphology interrelates the 
morphological classes of plant root, caulome for stem, phyllome for leaf and trichome for plant hair in generating coordinates transformed in 3D space to analyze plant architecture [1]. A plant, such as lettuce, has embryonic tissues that continually produce new tissues from its meristems for its development. Plant growth is a biological process in plants in which its structures experience maturity based on required inputs to its natural system. The morphological variation is primarily caused by positional effects, environmental effects, temperature variation exposure, and juvenility [2]. Plant proposes directional growth based on certain potent stimuli such as light, gravity, water, and physical contact for phototropism, gravitropism, hydrotropism, and thigmotropism. Lettuce plant development involves three distinct growth stages separated and ranged based on the number of days planted, namely, vegetative, head development, and harvest [3]. The vegetative growth stage starts from the sowing phase to the germination stage that usually takes 12 days. The Head development growth stage starts once the lettuce plant is transplanted as necessary, ranging from the third to sixth week of the plant life cycle. The harvest growth stage happens 45 to 65 days after sowing the lettuce seed. A comprehensive generic plant life cycle involves four stages, namely, seed germination, seed development, head development, and bolting harvest. The most conventional method to quantify lettuce's morphological development is using a scanning electron microscope [4]. Artificial light was instrumentalized to experiment morphological variations on lettuce [5]. The results showed that green, purple, and yellow artificial lights regulate biomass architecture, photosynthesis materialization, and the leaves' soluble sugar content [6]. Additional far-red (FR) light to mediate morphological and physiological indices of lettuce achieved 7 to $10 \%$ increase in photosynthetic activity [7] [8]. The use of color shade nets affected the morphological properties by inspecting its bolting, tip burn, rib discoloration, texture, and head shape [9].

Smart Farming is the implementation of advanced technologies and principles of precision agriculture to enhance the quantity and quality of agricultural yields through adjusting farm management and strategy [10]-[12]. It is otherwise conceptualized in different digitalization forms as decision agriculture [13], precision farming [14], digital agriculture [15], Agriculture 4.0 or numerical agriculture [16]. The sophistication of smart Farming involves sensor-based systems, telecommunications technologies for advanced networking, robotics and automation, autonomous data analytics tool, drones and swarm intelligence, artificial intelligence, satellites, and vision-based systems [17]-[19]. These advancements in farm operation systems provide a precise application of farm inputs to address production problems predominantly on water, energy, and chemical consumptions [20].

Emerging vision-based system applications in smart Farming have been used in lettuce growth monitoring with several unresolved tests that remain an open area for research. Convincingly, machine vision provided advanced and innovative approaches in plant growth monitoring and assessment [3] [21], [22]-[24]. Current innovations still characterize unsatisfactory solutions that challenge robust functionality testing, especially those employed in hydroponic and aquaponic environments. There is a very limited number of vision systems deployed in such occluding and complicated closed-environment agriculture (CEA) dealing with image segmentation on plant parts image explosion [25], segmentation of leaf affected with the disease [26], and feature extraction [27]. Most of the published studies presented lettuce planted on an open field and are soil-based [28] [29]. In this study, the researchers used lettuce images taken from a closed-environment smart farm employing nutrient film technique (NFT) hydroponics setup in an aquaponic system. As implied, the plant environment is differentiative in terms of the objects occluded on its middle ground and background and variations in illumination [30]. The resolution of the camera being used is also a substantial factor, together with scale invariance, as concurrent issues in vision systems [31]. Leaf are index (LAI) calculation, and grid counting was employed for non-destructive leaf area estimation (LAE) using a digital camera [32]. Leaf shape was computed based on the growth pattern model [33].

Superpixels technique provided advances in computer vision and image preprocessing [34], segmentation [35], semantic labelling [36], object detection and tracking [37] and classification [38]. A single superpixel holds extra spatial information than regular pixels; thus, it provides a compact, profound, and almost exact representation of the original image's abstraction. As superpixels, it uses lines and curves to create focal regions that are effective in computationally demanding tasks of 
segmentation. In this study, the superpixels technique is employed to segmentation the whole lettuce plant as single biomass.

Machine learning is an advanced artificial intelligence approach in making accurate predictions and classifications based on historical observations. It can be done using supervised and unsupervised learning. Supervised learning can be further divided into two, which are classification and regression algorithms. Under the classification algorithms are support vector machines, discriminant analysis, Naïve Bayes, nearest neighbor, and neural networks. Under regression, supervised learnings are linear regression, ensemble methods, decision trees, and neural networks.

Further details of the unsupervised machine learning are $\mathrm{K}$-means, $\mathrm{K}$-medoids, fuzzy $\mathrm{C}$-means, hierarchical, Gaussian mixture, hidden Markov model, and types of neural networks, such as selforganizing maps. Classification and identification are some of the most apparent and considerable aspects of supervised machine learning. For the most viable research, machine vision is paired with machine learning as it worked based on feature extraction of the most important characteristics of the object in focus. Machine vision and machine learning previously experimented in fresh-cut lettuce quality evaluation [39], change in growth features of planted lettuce [40], and plant disease detection [41] [42].

Despite the studies mentioned above, developments and innovations, image segmentation in vision system and plant growth monitoring, and stage identification with optimum accuracy is still open research. Plant science demands better morphological analysis better to understand the physiological and anatomical processes of plants. On the other hand, sustainable food production, particularly lettuce and other leafy vegetables, necessitates intelligent vision systems to manage farm management and strategies better.

This study's main objective is to identify the developmental growth stage of lettuce planted on hydroponics setup based on phytomorphological features. Specifically, this study aims to develop an algorithm on image segmentation through coupled superpixels and watershed transformations. In this research, morphological variation or geometric feature extractions used distance transformation, minima imposition, and blob operations. The optimized LDA, ANN, and SVM models were used to create classifying lettuce growth stage as vegetative, head development, and harvest.

\section{Method}

This section discusses the hydroponics setup, data description, and the three significant processes in determining a lettuce plant's growth stage, namely, image segmentation, morphological features extraction, and machine learning (Fig. 1).

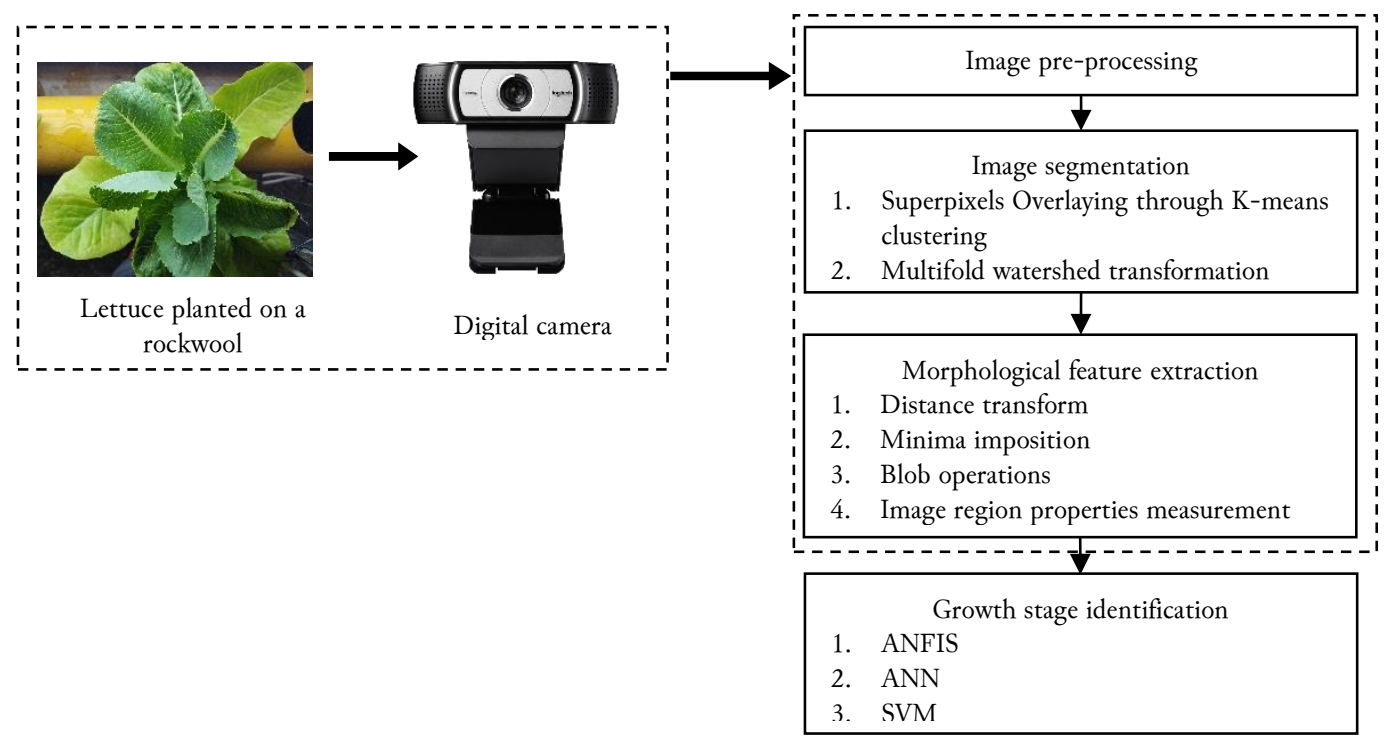

Fig. 1. Overall system architecture for lettuce growth stage identification 


\subsection{Growth bed setup}

The hydroponic setup in an aquaponic system is shown in Fig. 2 in which nutrient film technique was used as cultivation style. This setup is a horticultural technology that belongs to smart controlledenvironment agriculture. Four lettuce seeds are planted on a Rockwool with a size of 1 by 1 inch overlapped with a transparent plastic cup as a substitute to plant containers. This container is placed onto the hole at the top of the food-grade polyvinyl chloride (PVC) pipe. Pond water containing the concentrated nutrients from combined tilapia and carp effluents flow through the pipes. These nutrients' $\mathrm{pH}$ and electrical conductivity levels were maintained correctly based on the standard threshold for cultivating lettuce. The whole growth frame bed has three layers of growth bed. There is a total of 141 lettuces planted on it. Lettuce seeds were planted, germinated, and grown in this hydroponic setup without transplantation. Artificial lighting was employed using white LEDs, and no direct sunlight is allowed to expose the lettuce. The photoperiod was set to 24 hours for the vegetative stage, 21 hours to head development stage, and 12 hours for the harvest stage. Temperature is already maintained using exhaust fans.

\subsection{Dataset description}

Loose-leaf lettuce is the chosen type of lettuce to be cultivated. It can be easily harvested as a whole or by leaf, depending on its response to photosynthetic stimuli. The lettuce image set was gathered and collected in a smart lettuce farm in Rizal, Philippines, last August 2019 to October 2019. Lettuce images are taken using a digital camera. The system's image acquisition is vulnerable to some fundamental problems, such as noisy background, blurred image, Rockwool complementation, illumination, scale invariance, and chromatic intensity variations. Some of these problems are encountered and shown in Fig. 3. Moreover, 30 lettuce-captured images for every week from the first to tenth week of cultivation to monitor the distinct morphological growth of planted lettuce. There are 300 lettuce-captured images for the complete life cycle of lettuce plants that differ on crop size, plant dimensions, and its constituted morphological indices that are important in the vison-based system.

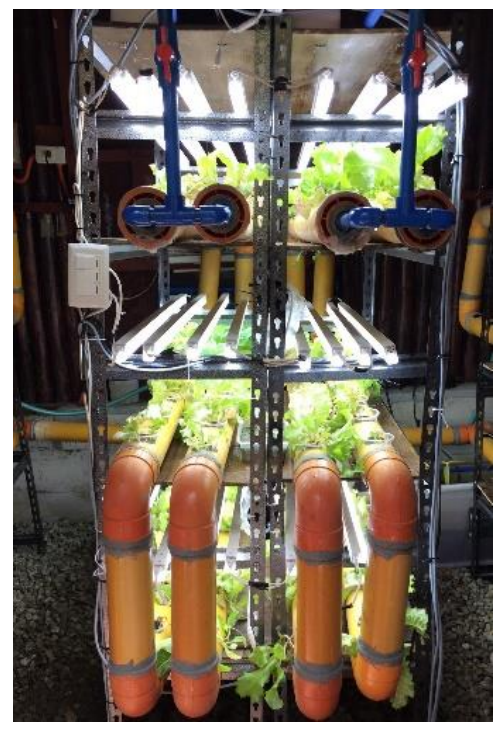

Fig. 2. Growth bed setup for lettuce cultivation

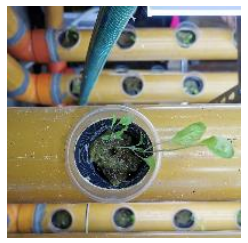

(a)

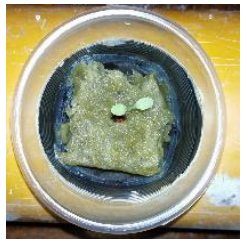

(c)

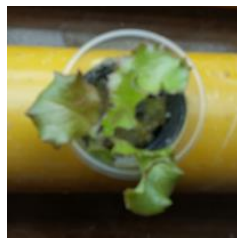

(b)

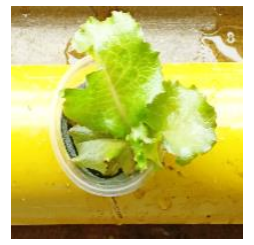

(d)
Fig. 3. Inherent vision problems for lettuce-captured images (a) noisy background (b) radial blurred capture (c) monotonous chromaticity to middle ground (d) illumination variations

There are three lettuce plant growth stages, namely, vegetative, head development, and harvest. The vegetative growth stage has 60 images distributed, as divided into 48 of the training images and 12 for the testing. The head development growth stage has 150 images distributed as 120 for the training and 30 for the testing. The lettuce ready to harvest stage has 90 images distributed as 72 for the training and 18 for the testing. Lettuce's maturity has no significance in the color specification so that gathered images can be green or turning violet. The dataset focuses on the morphological or geometrical features of the whole lettuce plant such as the number of leaves, biomass area and perimeter, convex area, convex 
hull $(\mathrm{CH})$ area and perimeter, major and minor axis length of the biomass, major axis length of the dominant leaf, length of plant skeleton, biomass compactness, convexity, and solidity, and the ratio of plant skeleton and perimeter. Thus, there are 300 rows by 14 columns of information on the image set.

\subsection{Image segmentation}

Image segmentation is a computer vision process of partitioning an $\mathrm{n}$-dimensional image into manifold pixel segments or image objects. This section is concerned with the detailed discussion of experiments done using the developed algorithm composed of coupled superpixels overlaying and multifold watershed transformation without thresholding to segment the whole lettuce plant, which is the image object in focus. Fig. 4 presents the detailed steps of the proposed image segmentation for the lettuce plant. Matlab language was used in developing the image processing program. Lettuce image passes through an image enhancement, which is chromatic aberration correction, as there is the presence of light intensity variation in the captured images. The resulting image is chromatically corrected and is passed on to RGB to CIELab conversion. Lab color space was chosen because it is the scientific color space that provides higher accuracy in the segmentation of complicated images. Superpixel overlays irregular geometrical pixels based on K-means on to the image for clustering colors. Next, image labeling was done to name the color clusters properly. Image filtering and smoothing provide additional enhancement to the image, especially to its contours. The next step is image object masking, which explodes each color cluster into different images. The superpixel computation, multiple watershed transformation, and other succeeding steps are presented separately in sections 2.3.1 and 2.3.2 for technical discussions.

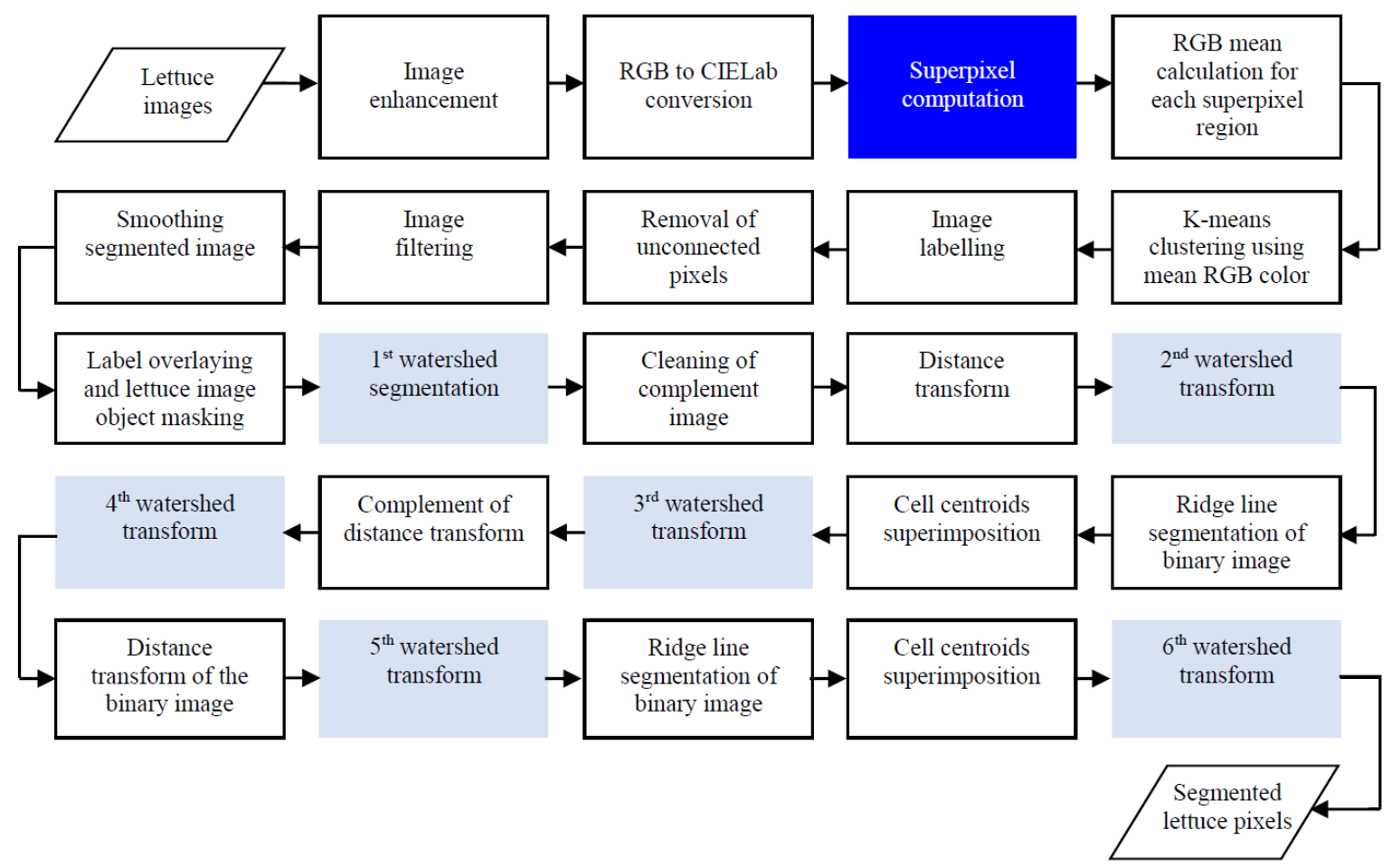

Fig. 4. Image processing used in this research

\subsubsection{Superpixels}

Segmentation of lettuce plants was performed based on color features using superpixels and K-means clustering. Superpixel is a computer vision technique of overlaying a superpixel region that is a geometrical section of an image that is larger than the regular pixel. It is rendered based on the uniformity of color and brightness. Simple linear iterative clustering (SLIC) algorithm is used to improve the segmentation based on color clustering superimposed by K-means. The lettuce-captured images undergo pre-processing of image enhancement and transform into its equivalent CIELab color space based on Eq. (1) to (6). The L, a, and b corresponds to luminosity, chromaticity present in the red- 
green axis, and chromaticity in the blue-yellow axis, respectively. The Lab color space is derived from the tristimulus of XYZ values, which are RGB extrapolations.

$$
\begin{aligned}
& X=0.412456 * R+0.357576 * G+0.180437 * B \\
& Y=0.212673 * R+0.715152 * G+0.072175 * B \\
& Z=0.019334 * R+0.715152 * G+0.072175 * B \\
& L=116 f(Y / 100)-16 \\
& a=500(f(X / 95.047)-f(Y / 100)) \\
& b=200(f(Y / 100)-f(Z / 108.883))
\end{aligned}
$$

The enhanced image is then overlaid with 50,100, 500, and 1000 superpixel regions, $n$, as shown in Fig. 5. It is used to experiment with the most suitable number of superpixels that can manually and visually identify the lettuce plant and counts the number of its leaves while processing in the shortest time possible. Table 1 shows that 1000 superpixel regions attained $99.33 \%$ positive visual identification on the number of leaves with an average processing time per image of $19.45 \mathrm{~s}$. However, as the number of superpixel regions decreases, the number of positive identifications also decreases but faster processing time. Despite the necessity to speed up the processing time for each image, it is more important to use a higher number of superpixel regions to attain higher accuracy. Thus, in this study, the employed number of superpixel regions is 1000 . Fig. 5 shows that SLIC sections the whole digital image into polygonal regions, unlike the conventional rectangular pixel, based on cluster regions on local spaces. This resolves the edge boundaries misalignment.
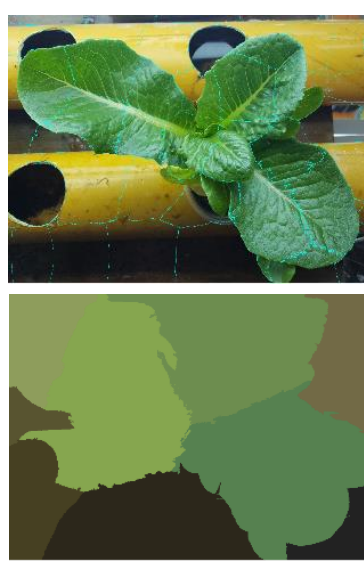

(a)
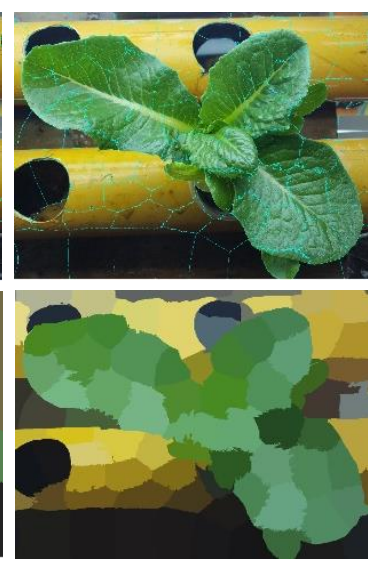

(b)
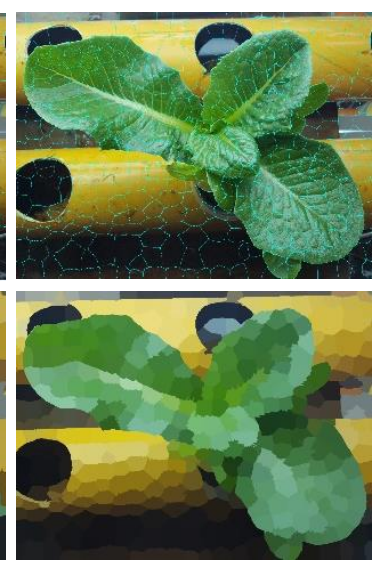

(c)

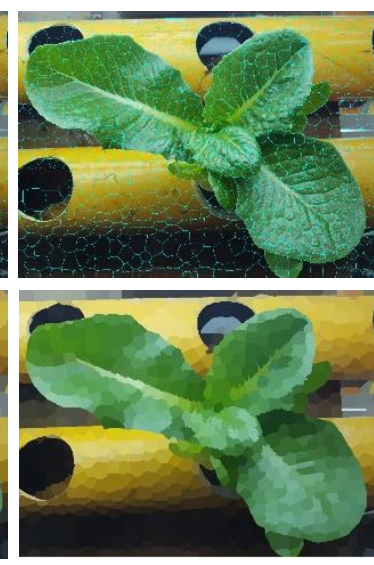

(d)

Fig. 5. Superpixel region boundaries overlaying and result for (a) $n=50$ (b) $n=100$ (c) $n=500$ (d) $n=1000$

Table 1. Number of superpixel region variations of visual identification and processing time

\begin{tabular}{cccc}
$\begin{array}{c}\text { No. of superpixel } \\
\text { regions }\end{array}$ & Positive identification & Negative identification & $\begin{array}{c}\text { Computing time per image } \\
(\mathbf{s})\end{array}$ \\
\hline 50 & 9 & 291 & 3.44 \\
100 & 211 & 89 & 5.92 \\
500 & 286 & 14 & 11.61 \\
1000 & 298 & 2 & 19.45 \\
\hline
\end{tabular}

Concepction II et al. (Lettuce growth stage identification based on phytomorphological variations using coupled color...) 
Next, K-means clustering with mean RGB color was implemented to similar group objects based on luminosity and chromaticity (Fig. 6) into several k mutually separated clusters. In this study, K-means clustering was set to repeat three times to avoid local minima so that object assignment is based on the nearest centroid for each cluster iteration. The subsequent superpixel RGB image undergoes image labeling. Based on experiments, the number of colors and the maximum number of masks for labeling that is suitable for the working image set are $15,10,5$, and 3 for week 1 , week 2 , weeks 3 to 7 , and weeks 8 to 10 of the lettuce life cycle, respectively. These dominant color preset variations correspond to the developmental changes of the lettuce plant's color feature as it matures. After masking, the image undergoes removal of disconnected regions with less than 1000 pixels and image filtering, retaining only the largest image object, which is the canopy of lettuce. Then, the segmented image is smoothened using a morphological flat structuring element.

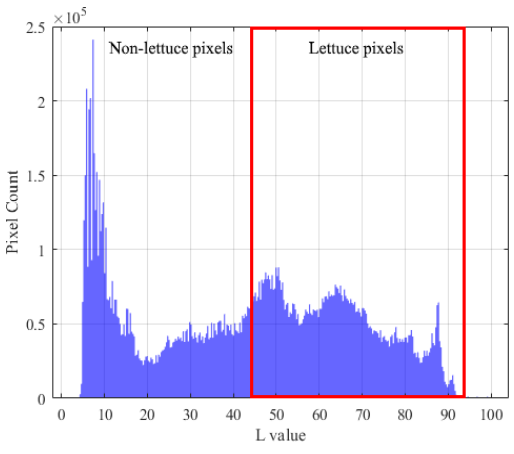

(a)

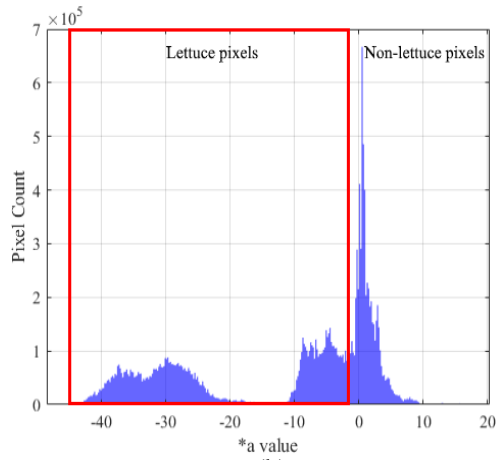

(b)

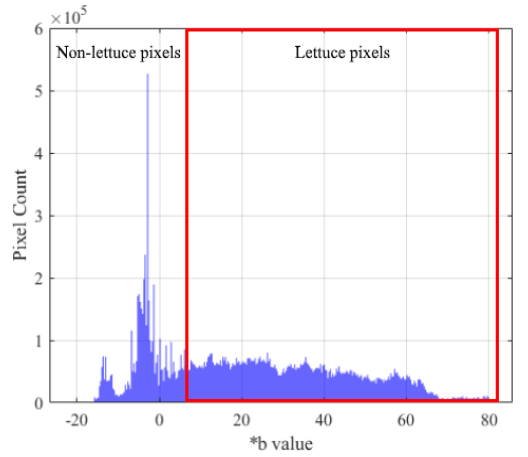

(c)

Fig. 6. CIELab thresholds after superpixel computation and K-mean clustering (a) L (b) a (c) b

\subsubsection{Multifold watershed transformation}

Multifold watershed transformation fine-tuned the superpixel segmentation by exploring the watershed ridgelines in a digital image. This concept is materialized when the algorithm considers light pixels in the image as high elevations and dark pixels for low elevations. In this study, a 6-level watershed transformation is employed to enhance the segmentation of lettuce further.

The smoothened segmented image is masked over the original image by label overlaying. Next, the overlaid 2D image was skeletonized by reducing the image object to a 1-pixel wide line. This protects the original topology and the Euler number of the image object in focus by isolating the centerline of the binary image. The skeletonized image undergoes edge localization based on pixel intensity using the Sobel method that detects edges for all quadrantal angles. Following the detection is the first watershed transformation that segments each lettuce leaf. Still, few small noises are present up to this stage; thus, image cleaning of objects less than 50 pixels is removed. The image is then subjected to the Euclidean distance transform of a single-pixel to its nearest neighboring positively valued pixel. After this, the image undergoes the second watershed transformation to fully segment the lettuce image through the Euclidean distance transform curves. Then, the thin lines are extended up to 80 pixels for cell centroids superimposition (CCS). CCS is performed through extended minima transform that computes the regional minima of constant intensity pixel boundaries. The Euclidean distance transformed image is altered by using morphological reconstruction imposition to attain minima on its boundary regions. Then, the third watershed transform is employed on the minima-imposed image. Another Euclidean distance transform was added to increase the solidity of the pixel boundaries. Then, the fourth watershed transformation subjects the complement distance transform the image by setting pixels to 0 for all sections outside the region of interest (ROI). This watershed transformation used 8-connect twodimensional connectivities. Another Euclidean distance transform was added to enhance the solidity of pixels further, and then, the fifth watershed transformation was employed. The ridgeline segmentation used the last binary image. For the last phase, a repetition of CCS was done but for the binary image. Lastly, the sixth watershed transformation was overlaid to mask the original image and produce the ROI's replica. The result of the sixth watershed transformation is the segmented lettuce pixels, as shown in Fig. 7. 


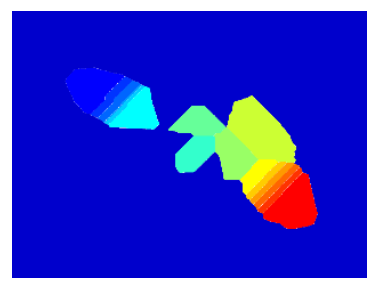

(a)

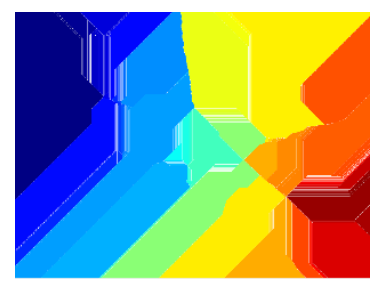

(e)

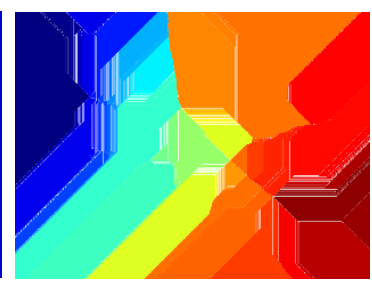

(b)

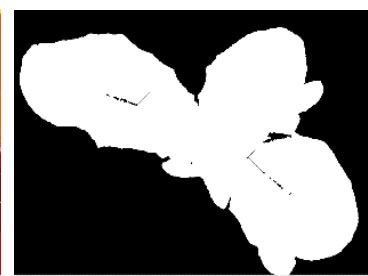

(f)

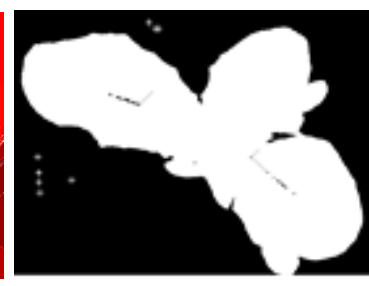

(c)

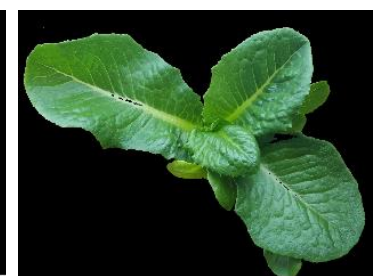

(g)

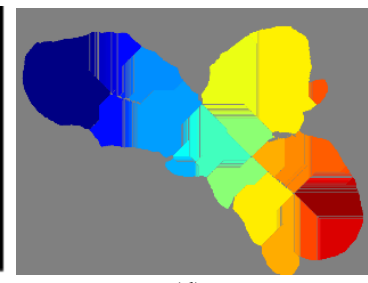

(d)

Fig. 7. Watershed transformations (a) first (b) second (c) third (d) fourth (e) fifth (f) sixth; and (g) segmented lettuce pixels

\subsection{Morphological feature extraction}

The morphological feature extraction employs the practicality of quantifying and observing the phytomorphological variations of lettuce plants based on the image morphological profile (IMP). Mathematically, morphological extraction is a method that performs erosion for opening the pixels and dilation for closing the pixels by applying it to pre-known regions called the structuring element (SE).

In this study, decision boundary feature extraction (DBFE) was employed instead of discriminant analysis feature extraction (DAFE) as it complements the boundary set by the previous step, which is dominantly done by superpixel ridge lines segmentation. Distance transform and minima imposition are both used to enhance the solidity of pixel geometry. After the pixels of ROI were segmented from the background, the masked image undergoes blob operations to remove extra objects that were not eliminated from image processing. This is to assure that the morphological features that will be extracted using region properties are true. Next, the image was employed with region properties computation for segmented leaf and whole biomass, and convex hull generation and flood-fill operation for whole biomass properties. The convex hull operation used the union method, which considers the foreground ROI, the lettuce pixels, as a single image object. It is otherwise termed as a convex envelope as it resembles a polygon that encloses all points derived from the image object ROI. This accounts that all points are located either on the interior or boundary of the enclosed convex hull. The 4-connected pixels value was set for flood-fill operation. The extracted phytomorphological features are the number of leaves, biomass area and perimeter, convex area, convex hull area and perimeter, major and minor axis length of the biomass, major axis length of the dominant leaf, length of plant skeleton, biomass compactness, convexity, and solidity, and the ratio of plant skeleton and perimeter.

\subsection{Machine learning models}

Machine learning models are analogous to functions that provide predictions from a given particular input. In the development of lettuce growth stage identification models, three approaches were used, namely: linear discriminant analysis (LDA), artificial neural network (ANN), and quadratic support vector machine (QSVM). For all models, 240 rows of input data are set for training and the other 60 are set for testing. These models were developed using the Matlab platform. LDA, ANN and QVSM were selected as these models maximizes its pattern recognition to discriminate classes of data which is necessary for this study. Linear and nonlinear clustering is the strength of these models.

\subsubsection{Linear discriminant analysis}

Linear discriminant analysis is a supervised machine learning model which generalizes the Fisher's linear discriminant used to recognize patterns for classification and dimensional reduction. It is otherwise 
known as normal discriminant analysis (NDA). It transforms one specific dependent variable or predictor as a linear combination with other predictors, thus characterizing distinct object classes. LDA also shows that it can separate linearly and non-linearly complicated data. It is a highly intelligent machine learning model for classification predictive problems. To prepare data for LDA, classify the problem, employ Gaussian distribution, remove outliers, and assume the same variance for each input variable. LDA uses Bayes theorem in estimating the demarcation of each class. The equation below shows the approximation of output class $(k)$ with input $(x)$ and the probability $(P)$ of specific data fitting to each class $(Y)$.

$$
P(Y=x \mid X=x)=(P \mid k * f k(x)) / \operatorname{sum}(P * f(x))
$$

In this study, linear discriminant analysis was furtherly employed such that the quadratic discriminant analysis (QDA) performed with an accuracy of $65.8 \%$, making it non-considerable for classification of this type of image set. The developed LDA has a full covariance structure with the deduction of principal component analysis (PCA) phase and 10-fold stratified cross-validation to avoid overfitting. The developed model has a prediction speed of approximately $1800 \mathrm{obs} / \mathrm{s}$ and a training time of $28.854 \mathrm{~s}$ for parallel pooling connection to 6 workers.

\subsubsection{Artificial neural network}

The artificial neural network is based on computational biology used to perform prediction, supervised and unsupervised classification, and pattern recognition [43] [44]. The raw inputs to the neural network work like synapses and are activated by a certain threshold that is then multiplied to its arbitrary weight [45]. Simply, ANN learns based on the input and output through backpropagation. A shallow neural network is considered the simplest type of ANN, which has a single hidden layer composed of multiple artificial neurons. This model initially goes through a training phase in which a certain percentage of the total dataset is inputted into its system for it to learn. The training phase constitutes the unoptimized state of the model. Then, by adjusting its hyperparameters such as the number of hidden layers and neurons, the model's learning classifier provides higher accuracy. An activation ( $a$ ) was set to correspond to the input layer. For the feedforward phase, a function $\mathrm{z}=\mathrm{w} \cdot \mathrm{a}+\mathrm{b}$ is computed for each layer. This is a measure of combined weight and activation with bias. The output error is then computed and backpropagated for each layer using the function z. Lastly, the output is then calculated using gradient descent or other classifiers for that neuron's weight and bias.

In this study, a three-layer feedforward neural network was developed with 14 input neurons on the first layer, 20 artificial neurons on the hidden second layer, and three artificial neurons on the output layer. A backpropagation algorithm was used for network learning. The number of hidden artificial neurons was characterized based on three network determinants, which are cross-entropy (CE) value, regression coefficient $(\mathrm{R})$, and processing time, which are factors in modeling the optimized neural network. Dual binary digit representation was used for output value representation.

\subsubsection{Quadratic support vector machine}

The support vector machine is considered a controlled machine learning model and a discriminative classifier that is basically used for regression and classification problems. It employs supervised learning by dividing an optimal hyperplane into a number of categories based on the labeled training data [46]. SVM determines the optimal solution in separating the hyperplane using its support vectors, which are the data points closest to the hyperplane. Usually, these closest data points exhibit the optimum location of separation for classification. The equation below forms the decision surface plane in discriminating data classes where $w^{T}$ is the transposed weight factor, $x$ is the input vector, and $b$ is bias. For QSVM, the function to be employed in discriminating the boundary of classes is quadratic; that is why the surface is a paraboloid with a single global minimum.

$$
w^{T} x+b=0
$$


In this study, the quadratic support vector machine (QSVM) was further employed because linear, cubic, fine Gaussian, medium Gaussian, and coarse gaussian SVM models attained lower training accuracy $82.1 \%, 85.8 \%, 83.8 \%, 85.4 \%$, and $75.8 \%$ respectively. The developed QSVM has quadratic kernel function, automatic kernel scale, box constraint level of 1 , one-vs-one multiclass method, and 10 -fold stratified cross-validation to avoid overfitting. The developed model has a prediction speed of approximately $1300 \mathrm{obs} / \mathrm{s}$ and a training time of $7.321 \mathrm{~s}$ for parallel pooling connected to 6 workers.

\section{Results and Discussion}

\subsection{Image segmentation}

Fig. 8 shows the sample results of the proposed segmentation for each lettuce growth stage. Region boundaries were computed using 1000 superpixels. Lettuce plant pixels were classified based on luminosity and chromaticity. It is noticeable that the image pertaining to the object in the ROI cluster for head development shows black pixels along the ridgeline of the largest leaf. The original green lettuce pixels were converted to black due to illumination. The lettuce's edge information in the original image was carefully clustered and preserved due to enough superpixel regions. The superpixels grouped the ROI into white pixels after the sixth watershed transformation, and the ROI lettuce image object was segmented.

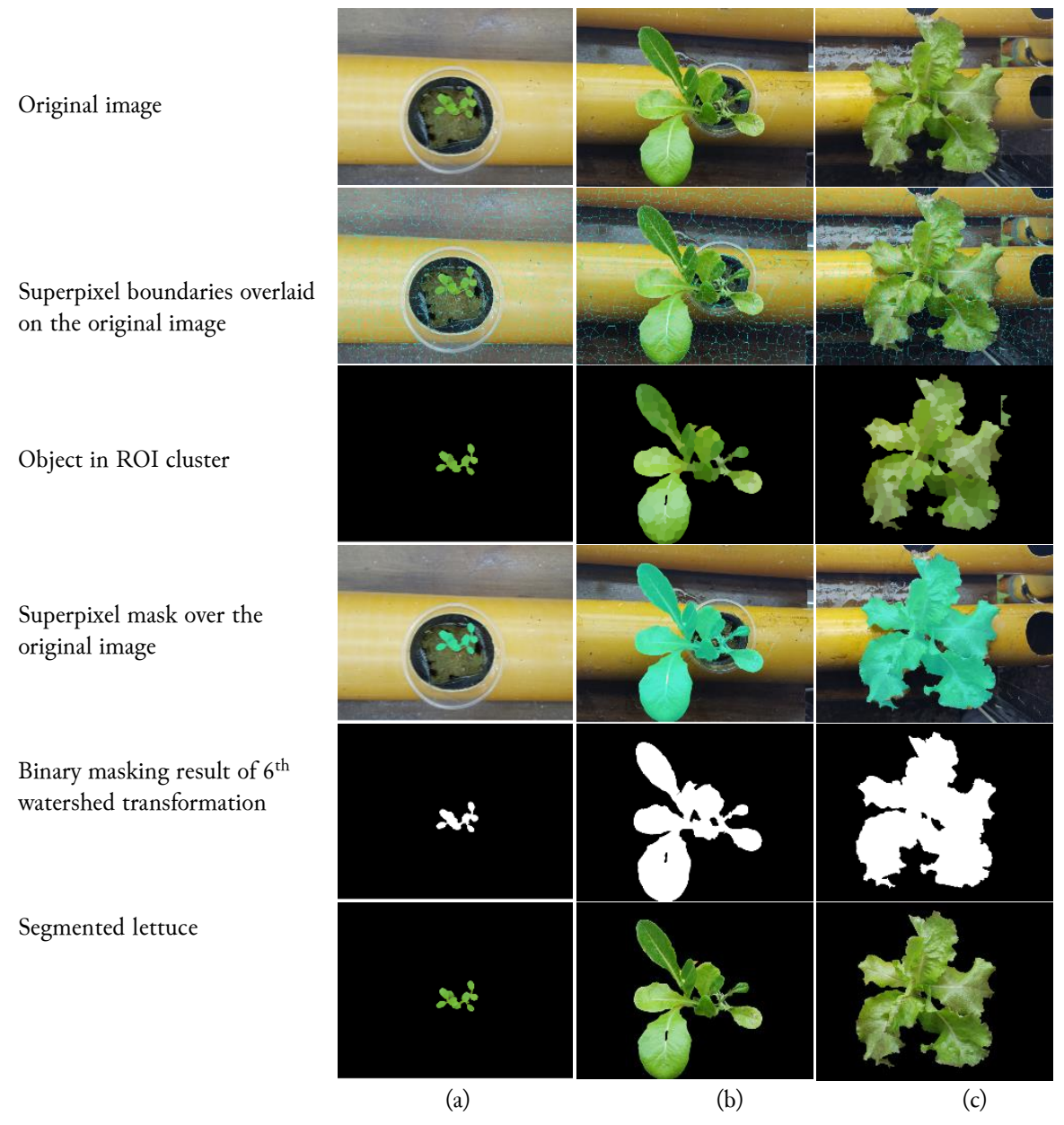

Fig. 8. Lettuce ROI segmentation for (a) vegetative (b) head development and (c) for harvest growth stages

\subsection{Phytomorphological feature extraction}

Table 2 summarized the descriptive statistics of phytomorphological features of the 300 sample images, where Q1 represents the vegetative to head development threshold for each feature, and Q3 
represents the threshold for head development to harvest the growth stage. The number of leaves is characterized by low weight in determining the growth stage as each Rockwool is planted with four lettuce seeds, and in the biological process of the plant, several seeds do not grow up to the genetically required number of leaves. Moreover, some lettuce experiences leaf diseases that frees some of its leaves from the stem during the development. However, biomass area and perimeter and biomass convex hull area and the perimeter are characterized as strong determinants in classifying lettuce growth stage.

Table 2. Descriptive statistics of phytomorphological features

\begin{tabular}{|c|c|c|c|c|c|c|c|c|}
\hline Feature & Mean & $\begin{array}{c}S E \\
\text { Mean }\end{array}$ & Std. Dev & Min. & Q1 & Median & Q3 & $\operatorname{Max}$ \\
\hline No. of leaves & 3.3800 & 0.0782 & 1.3546 & 1.0000 & 2.0000 & 3.0000 & 4.0000 & 9.0000 \\
\hline Biomass perimeter & 9046 & 458 & 7925 & 660 & 2227 & 6796 & 13927 & 37800 \\
\hline Biomass area & 1312979 & 98930 & 1713512 & 16741 & 127533 & 519330 & 1879725 & 8310300 \\
\hline Convex area & 2099381 & 154233 & 2671388 & 22917 & 172098 & 870540 & 3289225 & 12965000 \\
\hline Convex hull area & 2092241 & 153882 & 2665318 & 764 & 172098 & 870540 & 3178375 & 12965000 \\
\hline $\begin{array}{c}\text { Biomass convex hull } \\
\text { perimeter }\end{array}$ & 4716 & 204 & 3534 & 498 & 1675 & 3797 & 7149 & 14744 \\
\hline Biomas compactness & 0.016452 & 0.000642 & 0.011114 & 0.000013 & 0.008480 & 0.013372 & 0.020919 & 0.067303 \\
\hline Biomass solidity & 1.140 & 0.273 & 4.732 & 0.002 & 0.560 & 0.636 & 0.732 & 52.587 \\
\hline Biomass convexity & 1.7745 & 0.0280 & 0.4843 & 1.0122 & 1.4319 & 1.7024 & 2.0287 & 4.6876 \\
\hline $\begin{array}{l}\text { Dominant leaf major } \\
\text { axis length }\end{array}$ & 1625 & 375 & 6489 & 197 & 647 & 1111 & 1775 & 112720 \\
\hline $\begin{array}{c}\text { Biomass major axis } \\
\text { length }\end{array}$ & 1843.7 & 84.7 & 1466.2 & 196.9 & 668.9 & 1434.3 & 2690.4 & 7026.4 \\
\hline $\begin{array}{l}\text { Biomass minor axis } \\
\text { length }\end{array}$ & 930.5 & 41.7 & 722.4 & 76.7 & 319.1 & 718.0 & 1381.9 & 3201.8 \\
\hline $\begin{array}{l}\text { Length of biomass } \\
\text { skeleton }\end{array}$ & 23738 & 17802 & 308340 & 192 & 666 & 1353 & 2728 & 5151600 \\
\hline $\begin{array}{c}\text { Length of } \\
\text { skeleton/perimeter }\end{array}$ & 1.595 & 0.967 & 16.751 & 0.001 & 0.173 & 0.225 & 0.279 & 221.670 \\
\hline
\end{tabular}

\subsection{Optimized machine learning models}

Fig. 9 shows the normal discriminants of LDA based on biomass perimeter and biomass area, and biomass area and biomass minor axis length. The developed LDA model used 10-fold stratified crossvalidation instead of 3 to avoid overfitting which may result in poor classification performance. As seen in Fig. 9, the optimized LDA linearly separates three classes based on Bayes theorem.
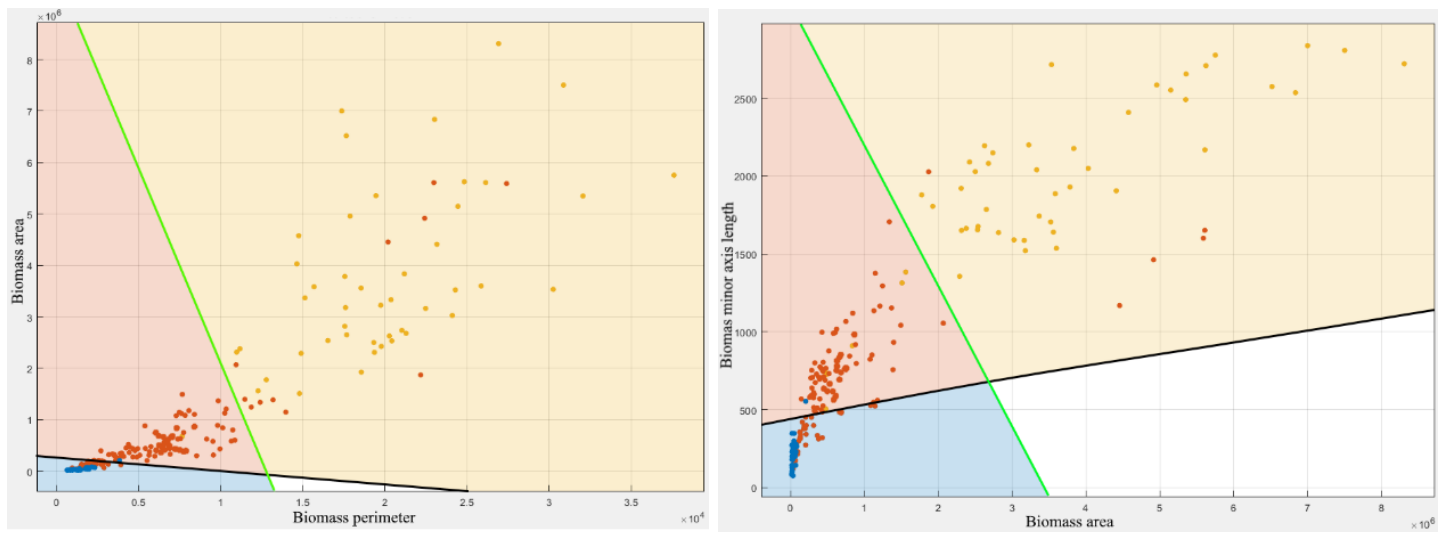

Fig. 9. Classification of lettuce growth stage using LDA 
Fig. 10 shows the train, validation, and test performance curves of the optimized neural network with a cross-entropy value of 0.069754 , indicating good network characteristics. The developed model adaptively converged on epoch 39, making the network suitable for classification based on inputted training data's learned characteristics. Cross-entropy, regression coefficient, and learning time are the concerned network parameters in determining the least computational neural network. Based on crossentropy, the 50 hidden artificial neurons performed best by having a CE value of 0.047175 and is followed by hidden artificial neurons of 20 with $\mathrm{CE}$ of 0.069754 . Based on the regression coefficient, the 900,90 , and 20 hidden artificial neurons performed best with values of $0.8626,0.84802$, and 0.84713 , respectively. Based on learning time, 10 and 20 hidden artificial neurons performed best with values of $1.05 \mathrm{~s}$ and $2 \mathrm{~s}$, respectively. Thus, making the 20 hidden artificial neurons most considerable to perform the least computational cost for classification.

Fig. 11 shows the quadratic support vector machine's discrimination on phytomorphological data of biomass perimeter and area, and biomass area and minor axis length. Quadratic curves are used to separate each class that is shown in different colors. The blue dots represent the phytomorphological characteristics of vegetative lettuce, red dots represent the lettuce under head development stage and orange dots represent the lettuce ready for harvest. As shown in Fig. 9, Fig. 10, and Fig. 11, the classification performance increased by adjusting its hyperparameters or determinant variables for all the developed machine learning models.

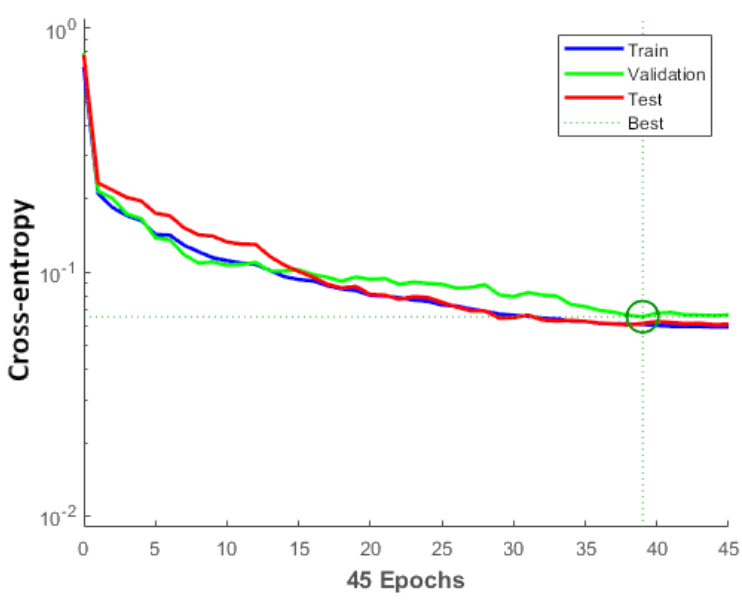

(a)
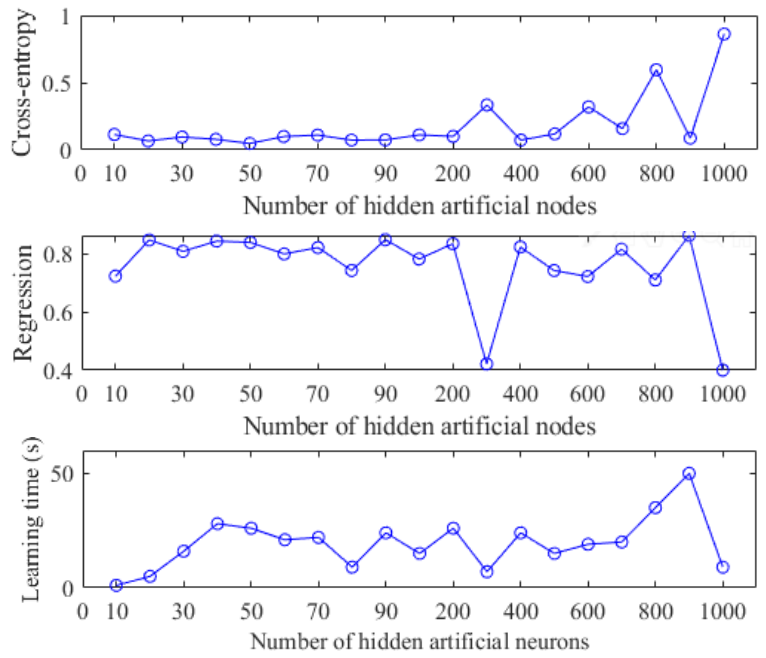

(b)

Fig. 10. Optimized neural network (a) performance plot of developed 3-layer ANN with 20 hidden artificial neurons (b) network performance determinants
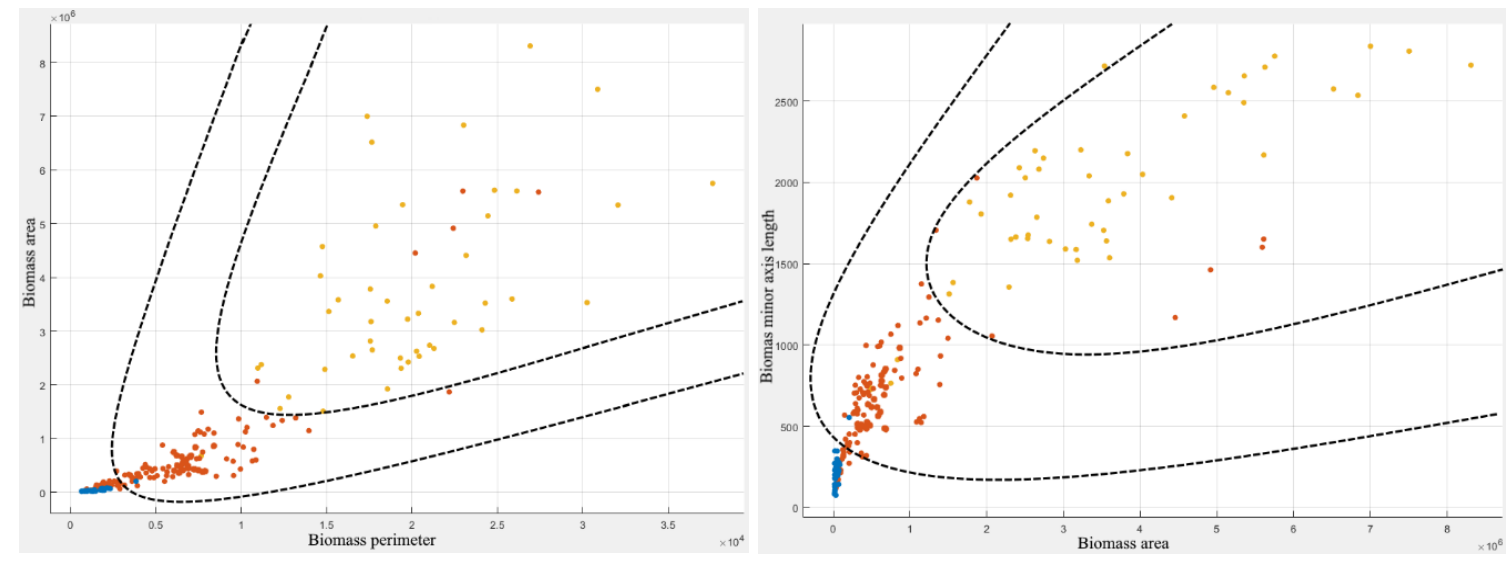

Fig. 11. Classification of lettuce growth stage using QSVM 


\subsection{Lettuce growth stage identification}

By using the three optimized machine learning models, Table 3 presents the classification scores on each lettuce growth stage during the testing phase. For the vegetative growth stage, ANN is the only machine learning model, which misclassified to the given set of 14-column phytomorphological data with a negative score of 4 . The ANN model also misclassified the head development data set twice as harvest data set, and the same with for harvest data set. The LDA model perfectly classified the vegetative data set but attained the highest misclassification rate for the harvest growth stage with a misclassification score of 7 out of 18. On the other hand, QSVM perfectly classified the vegetative data set and performed the lowest misclassification score of 2 out of 18 for the harvest growth stage. The three models easily classified vegetative lettuce due to the significant difference of phytomorphological numerical values as this stage only resembles the seed leaf and the lettuce 2-true-leaves stage.

Table 3. Classification scores for the testing phase resulted from using the optimized machine learning models

\begin{tabular}{ccccccc}
\hline \multirow{2}{*}{ Model } & \multicolumn{3}{c}{ Correct } & \multicolumn{3}{c}{ Incorrect } \\
\cline { 2 - 6 } & Vegetative & $\begin{array}{c}\text { Head } \\
\text { Development }\end{array}$ & Harvest & Vegetative & $\begin{array}{c}\text { Head } \\
\text { Development }\end{array}$ & Harvest \\
\hline LDA & 12 & 25 & 11 & 0 & 5 & 7 \\
ANN & 8 & 28 & 16 & 4 & 2 & 2 \\
QSVM & 12 & 25 & 16 & 0 & 5 & 2 \\
\hline
\end{tabular}

Table 4 shows the listing of the training and testing accuracy characterized by each optimized machine learning model. The ANN model was performed as the most accurate model during the training phase, with $90.00 \%$ correct classification of lettuce growth stages out of 240 samples. Even though all models meet the desired $80 \%$ and above accuracy during the training phase, the LDA model failed to maintain it during the testing phase resulting in $60.00 \%$. The ANN model is still above the desired $80 \%$ accuracy during the testing phase but is diminished by $5 \%$. However, the QSVM even has higher accuracy during the testing phase, resulting in $88.33 \%$, making it the best-optimized machine learning model. This model is consistent in providing correct classifications supported by its performance and accuracy. The SVM is not overfitted due to the configuration of 10 -fold stratified cross-validation.

Table 4. Accuracy performance of lettuce growth stage classification models using machine learning

\begin{tabular}{cccccccc}
\hline & & \multicolumn{2}{c}{ Training } & \multicolumn{3}{c}{ Testing Using Optimized Model } \\
\cline { 3 - 7 } Model & Splitting & $\begin{array}{c}\text { No. of } \\
\text { Samples }\end{array}$ & Accuracy & $\begin{array}{c}\text { No. of } \\
\text { Samples }\end{array}$ & Correct & Incorrect & Accuracy \\
\hline LDA & $80-20$ & 240 & $80.42 \%$ & 60 & 48 & 12 & $60.00 \%$ \\
ANN & $80-20$ & 240 & $90.00 \%$ & 60 & 51 & 9 & $85.00 \%$ \\
QSVM & $80-20$ & 240 & $87.90 \%$ & 60 & 53 & 7 & $88.33 \%$ \\
\hline
\end{tabular}

Summarized in Table 5 is the comparison of the three models, LDA, ANN, and QSVM, in terms of accuracy, precision, recall, and f1-score. The models were trained by adjusting their respective hyperparameters, such as adding neurons in ANN's stand. It can be seen from the above-presented table that all models exhibit consistency from unoptimized state to the optimized state. This entails the none of the models demonstrates overfitting.

Table 5. Algorithm comparison based on selected evaluation metrics

\begin{tabular}{ccccccccc}
\hline \multirow{2}{*}{ Model } & \multicolumn{3}{c}{ Unoptimized Model } & \multicolumn{5}{c}{ Optimized Model } \\
\cline { 2 - 9 } & Accuracy & Precision & Recall & f1-score & Accuracy & Precision & Recall & $f 1$-score \\
\hline LDA & $80.42 \%$ & $73.68 \%$ & $65.12 \%$ & $69.14 \%$ & $60.00 \%$ & $80.00 \%$ & $88.89 \%$ & $84.21 \%$ \\
ANN & $90.00 \%$ & $84.44 \%$ & $71.70 \%$ & $77.55 \%$ & $85.00 \%$ & $85.00 \%$ & $92.73 \%$ & $88.70 \%$ \\
QSVM & $87.90 \%$ & $57.50 \%$ & $60.53 \%$ & $58.97 \%$ & $88.33 \%$ & $88.33 \%$ & $94.64 \%$ & $91.38 \%$ \\
\hline
\end{tabular}




\section{Conclusion}

Different algorithms for classifying lettuce growth stages into vegetative, head development and for harvest using the combinations of color SLIC superpixels and 6-fold watershed transformation and machine learning models are proposed in this paper. Based on the results of image ROI segmentation, the methodology attained enhanced segmentation of lettuce plant and background pixels separately. Ten phytomorphological features were geometrically extracted, namely, number of leaves, biomass area and perimeter, convex area, convex hull $(\mathrm{CH})$ area and perimeter, major, and minor axis length of the biomass, major axis length of the dominant leaf, length of plant skeleton. Additional four phytomorphological profile indices were computed out of the extracted features, namely, biomass compactness, convexity, solidity, and plant skeleton, and perimeter ratio. The optimized LDA model is very unstable, as its accuracy diminished by more than $20 \%$ during the testing phase. The 3 -layer ANN model with 20 hidden artificial neurons registered the highest accuracy of $90 \%$ during the training phase, but the optimized QSVM is considered as the most stable model with $87.90 \%$ and $88.33 \%$ training and testing accuracy, respectively. Thus, making QSVM the best machine learning model to implement lettuce growth stage classification based on phytomorphological features. Optimizing these machine learning models increased the accuracy of QSVM classification by $0.43 \%$. On the other hand, there is a significant decrease in classification accuracy in LDA and ANN models: $-20.42 \%$ and $-5 \%$, respectively. QVSM is the most consistent model from unoptimized to optimized state based on accuracy, precision, recall, and f1-score. This enhancement of QSVM provides greater integrity in identifying the correct lettuce life cycle stage. Future works include the further segmentation of leaves from the stem and the introduction of feature reduction to optimize computational time. Moreover, an additional image set must be used to increase the accuracy of the system further.

\section{Acknowledgment}

The authors would like to thank the Engineering Research and Development for Technology (ERDT) of the Department of Science and Technology (DOST) of the Philippines and the Intelligent Systems Laboratory of the De La Salle University for the granted supports.

\section{Declarations}

Author contribution. All authors contributed equally to the main contributor to this paper. All authors read and approved the final paper.

Funding statement. None of the authors have received any funding or grants from any institution or funding body for the research.

Conflict of interest. The authors declare no conflict of interest.

Additional information. No additional information is available for this paper.

\section{References}

[1] A. Conn, U. V. Pedmale, J. Chory, and S. Navlakha, "High-Resolution Laser Scanning Reveals Plant Architectures that Reflect Universal Network Design Principles," Cell Syst., vol. 5, no. 1, pp. 53-62.e3, Jul. 2017, doi: 10.1016/j.cels.2017.06.017.

[2] J. Jin et al., "An Arabidopsis Transcriptional Regulatory Map Reveals Distinct Functional and Evolutionary Features of Novel Transcription Factors," Mol. Biol. Evol., vol. 32, no. 7, pp. 1767-1773, Jul. 2015, doi: 10.1093/molbev/msv058.

[3] P. J. M. Loresco, I. C. Valenzuela, and E. P. Dadios, "Color Space Analysis Using KNN for Lettuce Crop Stages Identification in Smart Farm Setup," in TENCON 2018 - 2018 IEEE Region 10 Conference, 2018, pp. 2040-2044, doi: 10.1109/TENCON.2018.8650209.

[4] W. de Bruin, C. F. van der Merwe, Q. Kritzinger, R. M. S. Bornman, and L. Korsten, "Morphological characterisation of lettuce plasma membrane ultrastructure and vesicle formation caused by nonylphenol: A scanning electron microscopy study," South African J. Bot., vol. 111, pp. 176-181, Jul. 2017, doi: 10.1016/j.sajb.2017.03.027. 
[5] Z. Yan, D. He, G. Niu, and H. Zhai, "Evaluation of growth and quality of hydroponic lettuce at harvest as affected by the light intensity, photoperiod and light quality at seedling stage," Sci. Hortic. (Amsterdam)., vol. 248, pp. 138-144, Apr. 2019, doi: 10.1016/j.scienta.2019.01.002.

[6] H. Liu, Y. Fu, D. Hu, J. Yu, and H. Liu, "Effect of green, yellow and purple radiation on biomass, photosynthesis, morphology and soluble sugar content of leafy lettuce via spectral wavebands "knock out,"” Sci. Hortic. (Amsterdam)., vol. 236, pp. 10-17, Jun. 2018, doi: 10.1016/j.scienta.2018.03.027.

[7] J. Zou et al., "Morphological and physiological properties of indoor cultivated lettuce in response to additional far-red light," Sci. Hortic. (Amsterdam)., vol. 257, p. 108725, Nov. 2019, doi: 10.1016/j.scienta.2019.108725.

[8] Q. Meng and E. S. Runkle, "Far-red radiation interacts with relative and absolute blue and red photon flux densities to regulate growth, morphology, and pigmentation of lettuce and basil seedlings," Sci. Hortic. (Amsterdam)., vol. 255, pp. 269-280, Sep. 2019, doi: 10.1016/j.scienta.2019.05.030.

[9] S. Z. Ilić et al., "Light modification by color nets improve quality of lettuce from summer production," Sci. Hortic. (Amsterdam)., vol. 226, pp. 389-397, Dec. 2017, doi: 10.1016/j.scienta.2017.09.009.

[10] J. E. Relf-Eckstein, A. T. Ballantyne, and P. W. B. Phillips, "Farming Reimagined: A case study of autonomous farm equipment and creating an innovation opportunity space for broadacre smart farming," NJAS - Wageningen J. Life Sci., vol. 90-91, p. 100307, Dec. 2019, doi: 10.1016/j.njas.2019.100307.

[11] A. A. Adenle, H. Azadi, and J. Arbiol, "Global assessment of technological innovation for climate change adaptation and mitigation in developing world," J. Environ. Manage., vol. 161, pp. 261-275, Sep. 2015, doi: 10.1016/j.jenvman.2015.05.040.

[12] V. Blok and B. Gremmen, "Agricultural Technologies as Living Machines: Toward a Biomimetic Conceptualization of Smart Farming Technologies," Ethics, Policy Environ., vol. 21, no. 2, pp. 246-263, May 2018, doi: 10.1080/21550085.2018.1509491.

[13] A. Zhang, I. Baker, E. Jakku, and R. Llewellyn, "Accelerating precision agriculture to decision agriculture: The needs and drivers for the present and future of digital agriculture in Australia," 2017. Available at: Google Scholar

[14] C. Eastwood, M. Ayre, R. Nettle, and B. Dela Rue, "Making sense in the cloud: Farm advisory services in a smart farming future," NJAS - Wageningen J. Life Sci., vol. 90-91, p. 100298, Dec. 2019, doi: 10.1016/j.njas.2019.04.004.

[15] M. Shepherd, J. A. Turner, B. Small, and D. Wheeler, "Priorities for science to overcome hurdles thwarting the full promise of the 'digital agriculture' revolution," J. Sci. Food Agric., vol. 100, no. 14, pp. 5083-5092, Nov. 2020, doi: 10.1002/jsfa.9346.

[16] V. Bellon Maurel and C. Huyghe, "Putting agricultural equipment and digital technologies at the cutting edge of agroecology," OCL, vol. 24, no. 3, p. D307, May 2017, doi: 10.1051/oc1/2017028.

[17] J. Doshi, T. Patel, and S. kumar Bharti, "Smart Farming using IoT, a solution for optimally monitoring farming conditions," Procedia Comput. Sci., vol. 160, pp. 746-751, 2019, doi: 10.1016/j.procs.2019.11.016.

[18] L. Klerkx, E. Jakku, and P. Labarthe, "A review of social science on digital agriculture, smart farming and agriculture 4.0: New contributions and a future research agenda," NJAS - Wageningen J. Life Sci., vol. 9091, p. 100315, Dec. 2019, doi: 10.1016/j.njas.2019.100315.

[19] D. Glaroudis, A. Iossifides, and P. Chatzimisios, "Survey, comparison and research challenges of IoT application protocols for smart farming," Comput. Networks, vol. 168, p. 107037, Feb. 2020, doi: 10.1016/j.comnet.2019.107037.

[20] A. T. Balafoutis et al., "Smart Farming Technologies - Description, Taxonomy and Economic Impact," 2017, pp. 21-77. doi: 10.1007/978-3-319-68715-5_2

[21] I. C. Valenzuela et al., "Quality assessment of lettuce using artificial neural network," in 2017IEEE 9th International Conference on Humanoid, Nanotechnology, Information Technology, Communication and Control, Environment and Management (HNICEM), 2017, pp. 1-5, doi: 10.1109/HNICEM.2017.8269506. 
[22] X. A. P. Calangian et al., "Vision-based Canopy Area Measurements," in 2018 IEEE 10th International Conference on Humanoid, Nanotechnology, Information Technology, Communication and Control, Environment and Management (HNICEM), 2018, pp. 1-4, doi: 10.1109/HNICEM.2018.8666251.

[23] Y. Fang, X. Wang, P. Shi, C. Lin, and R. Zhai, "Automatic identification of two growth stages for rapeseed plant: Three leaf and four leaf stage," in 2015 Fourth International Conference on Agro-Geoinformatics (Agrogeoinformatics), 2015, pp. 148-153, doi: 10.1109/Agro-Geoinformatics.2015.7248125.

[24] W. Gelard, A. Herbulot, M. Devy, and P. Casadebaig, "3D Leaf Tracking for Plant Growth Monitoring," in 2018 25th IEEE International Conference on Image Processing (ICIP), 2018, pp. 3663-3667, doi: 10.1109/ICIP.2018.8451553.

[25] M. P. Rico-Fernández, R. Rios-Cabrera, M. Castelán, H.-I. Guerrero-Reyes, and A. Juarez-Maldonado, "A contextualized approach for segmentation of foliage in different crop species," Comput. Electron. Agric., vol. 156, pp. 378-386, Jan. 2019, doi: 10.1016/j.compag.2018.11.033.

[26] X. Guo, M. Zhang, and Y. Dai, "Image of Plant Disease Segmentation Model Based on Pulse Coupled Neural Network with Shuffle Frog Leap Algorithm," in 2018 14th International Conference on Computational Intelligence and Security (CIS), 2018, pp. 169-173, doi: 10.1109/CIS2018.2018.00044.

[27] P. J. Loresco, A. Bandala, A. Culaba, and E. Dadios, "Computer vision performance metrics evaluation of object detection based on Haar-like, HOG and LBP features for scale-invariant lettuce leaf area calculation," Int. J. Eng. Technol., vol. 7, no. 4, pp. 4866-4872, 2018, doi: 10.14419/ijet.v7i4.26071

[28] D. G. Fernández-Pacheco, D. Escarabajal-Henarejos, A. Ruiz-Canales, J. Conesa, and J. M. MolinaMartínez, "A digital image-processing-based method for determining the crop coefficient of lettuce crops in the southeast of Spain," Biosyst. Eng., vol. 117, pp. 23-34, Jan. 2014, doi: 10.1016/j.biosystemseng.2013.07.014.

[29] D. Escarabajal-Henarejos, J. M. Molina-Martínez, D. G. Fernández-Pacheco, F. Cavas-Martínez, and G. García-Mateos, "Digital photography applied to irrigation management of Little Gem lettuce," Agric. Water Manag., vol. 151, pp. 148-157, Mar. 2015, doi: 10.1016/j.agwat.2014.08.009.

[30] R. Bhagwat and Y. Dandawate, "Indian plant species identification under varying illumination and viewpoint conditions," in 2016 Conference on Advances in Signal Processing (CASP), 2016, pp. 469-473, doi: 10.1109/CASP.2016.7746217.

[31] P. J. M. Loresco and E. P. Dadios, "A scale-invariant lettuce leaf area calculation using machine vision and knowledge-based methods,” Int. J. Eng. Technol., vol. 7, no. 4, pp. 4880-4885, 2018, doi: 10.14419/ijet.v7i4.26553

[32] H. Hajjdiab and A. Obaid, "A vision-based approach for nondestructive leaf area estimation," in 2010 The 2nd Conference on Environmental Science and Information Application Technology, 2010, pp. 53-56, doi: 10.1109/ESIAT.2010.5568973.

[33] Y. Hong, Z. Chen, and J. Qiu, "Classification of leaf shapes and estimation of leaf mass," in 2012 International Conference on Green and Ubiquitous Technology, 2012, pp. 167-171, doi: 10.1109/GUT.2012.6344176.

[34] S. Zhang, H. Wang, W. Huang, and Z. You, "Plant diseased leaf segmentation and recognition by fusion of superpixel, K-means and PHOG," Optik (Stuttg)., vol. 157, pp. 866-872, Mar. 2018, doi: 10.1016/j.ijleo.2017.11.190.

[35] Y. Shen, T. Ai, W. Li, M. Yang, and Y. Feng, "A polygon aggregation method with global feature preservation using superpixel segmentation," Comput. Environ. Urban Syst., vol. 75, pp. 117-131, May 2019, doi: 10.1016/j.compenvurbsys.2019.01.009.

[36] I. Jebari and D. Filliat, "Color and Depth-Based Superpixels for Background and Object Segmentation," Procedia Eng., vol. 41, pp. 1307-1315, 2012, doi: 10.1016/j.proeng.2012.07.315.

[37] K. Fu, C. Gong, J. Yang, Y. Zhou, and I. Yu-Hua Gu, "Superpixel based color contrast and color distribution driven salient object detection," Signal Process. Image Commun., vol. 28, no. 10, pp. 1448-1463, Nov. 2013, doi: 10.1016/j.image.2013.07.005. 
[38] L. Zhang, B. Verma, and D. Stockwell, "Spatial contextual superpixel model for natural roadside vegetation classification,” Pattern Recognit., vol. 60, pp. 444-457, Dec. 2016, doi: 10.1016/j.patcog.2016.05.013.

[39] B. Pace, M. Cefola, P. Da Pelo, F. Renna, and G. Attolico, "Non-destructive evaluation of quality and ammonia content in whole and fresh-cut lettuce by computer vision system," Food Res. Int., vol. 64, pp. 647-655, Oct. 2014, doi: 10.1016/j.foodres.2014.07.037.

[40] M. Yazdi, M. Kolahi, E. Mohajel Kazemi, and A. Goldson Barnaby, "Study of the contamination rate and change in growth features of lettuce (Lactuca sativa Linn.) in response to cadmium and a survey of its phytochelatin synthase gene," Ecotoxicol. Environ. Saf., vol. 180, pp. 295-308, Sep. 2019, doi: 10.1016/j.ecoenv.2019.04.071.

[41] J. G. A. Barbedo, "Detection of nutrition deficiencies in plants using proximal images and machine learning: A review," Comput. Electron. Agric., vol. 162, pp. 482-492, Jul. 2019, doi: 10.1016/j.compag.2019.04.035.

[42] H. Mao, H. Gao, X. Zhang, and F. Kumi, "Nondestructive measurement of total nitrogen in lettuce by integrating spectroscopy and computer vision," Sci. Hortic. (Amsterdam)., vol. 184, pp. 1-7, Mar. 2015, doi: 10.1016/j.scienta.2014.12.027.

[43] R. S. Concepcion and L. C. Ilagan, "Application of Hybrid Soft Computing for Classification of Reinforced Concrete Bridge Structural Health Based on Thermal-Vibration Intelligent System Parameters," in 2019 IEEE 15th International Colloquium on Signal Processing \& Its Applications (CSPA), 2019, pp. 207-212, doi: 10.1109/CSPA.2019.8696007.

[44] R. S. Concepcion et al., "Alertness and Mental Fatigue Classification Using Computational Intelligence in an Electrocardiography and Electromyography System with Off-Body Area Network,” 2020, pp. 153-169, doi: 10.1007/978-3-030-20904-9_12

[45] R. S. Concepcion II, P. J. M. Loresco, R. A. R. Bedruz, E. P. Dadios, S. C. Lauguico, and E. Sybingco, "Trophic state assessment using hybrid classification tree-artificial neural network," Int. J. Adv. Intell. Informatics, vol. 6, no. 1, p. 46, Mar. 2020, doi: 10.26555/ijain.v6i1.408.

[46] I. Dagher, “Quadratic kernel-free non-linear support vector machine,” J. Glob. Optim., vol. 41, no. 1, pp. 15-30, May 2008, doi: 10.1007/s10898-007-9162-0. 